\title{
Cáncer de mama: una visión general
}

\section{Breast cancer: an overview}

\author{
Joaquín Palmero Picazo, ${ }^{*}$ Jareth Lassard Rosenthal, * \\ Leslie Aylin Juárez Aguilar,* Carlos Alonso Medina Núñez*
}

Citar como: Palmero PJ, Lassard RJ, Juárez ALA, Medina NCA. Cáncer de mama: una visión general. Acta Med Grupo Angeles. 2021; 19 (3): 354-360. https://dx.doi.org/10.35366/101727

\section{Resumen}

El cáncer de mama es la neoplasia más prevalente en población femenina, tan sólo en México se cuenta con una incidencia anual de 38.4 por 100,000 mujeres, donde el costo anual de cada paciente es de $\$ 110,459$.00 pesos, causando un gran impacto en el sistema de salud. Objetivos: Describir la etiopatogenia, epidemiología, cuadro clínico, clasificación y tamizaje actualizado de esta patología. Material y métodos: Se realizó una búsqueda sistemática en las bases de datos PubMed, Web of Science y EBSCO, utilizando los términos de búsqueda: breast cancer, pathology, therapy, prevention and control; con el operador booleano "and". Se seleccionaron los artículos que tuvieran un aporte relevante a este trabajo, obteniendo 30 artículos como muestra final. Resultados: La fisiopatología involucra vías como PI3K/AKT y RAS/MEK/ERK, y la presencia de receptores hormonales. El tamizaje se lleva a cabo mediante el examen clínico y la mastografía, mientras que el diagnóstico definitivo es mediante técnicas de biopsia. Por último, existen cinco caminos de tratamiento: cirugía, radioterapia, quimioterapia, hormonoterapia y biológicos, cada uno con sus indicaciones puntuales. Conclusiones: Es vital la capacitación del personal médico sobre esta patología, así como el desarrollo de un plan estratégico eficiente encaminado a una detección temprana oportuna.

Palabras clave: Cáncer de mama, patología, terapia, prevención y control.

\footnotetext{
* Médico pasante de Servicio Social de la carrera de Médico Cirujano, Facultad de Ciencias de la Salud, Universidad Anáhuac México Norte. México.
}

Correspondencia: Joaquín Palmero Picazo

Correo electrónico: joaquin.palmero@anahuac.mx

Aceptado: 19-01-2021.

www.medigraphic.com/actamedica

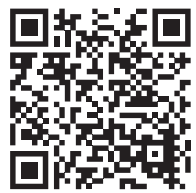

\section{Abstract}

Breast cancer is the most prevalent neoplasm in the female population. In Mexico alone there is an annual incidence of 38.4 per 100,000 women, where the annual cost of each patient is $\$ 110,459.00$ Mexican pesos, causing a great impact on the health system. Objectives: To describe the etiopathogeny, epidemiology, clinical picture, classification and updated screening of this pathology. Material and methods: A systematic search was carried out in the databases PubMed, Web of Science and EBSCO, using the search terms: breast cancer, pathology, therapy, prevention and control; with the Boolean operator "and". Selecting the articles that had a relevant contribution to this work. We obtained 30 articles as a final sample. Results: The physiopathology involves pathways such as PI3K/AKT and RAS/MEK/ERK, and the presence of hormone receptors. Screening is carried out by clinical examination and mastography, while the definitive diagnosis is made by biopsy techniques. Finally, there are five treatment paths: surgery, radiotherapy, chemotherapy, hormone therapy and biological, each with its specific indications. Conclusions: It is vital the training of medical personnel on this pathology, as well as the development of an efficient strategic plan aimed at early detection.

Keywords: Breast cancer, pathology, therapy, prevention and control.

\section{INTRODUCCIÓN}

El cáncer de mama fue descrito por primera vez en el Papiro Edwin Smith, entre el año 3,000 y 2,500 a.C. En 1882, William Halsted realizó la primera mastectomía, y desde ese momento, hitos como el vaciamiento ganglionar de cuello y mediastino anterior de Handley en 1901, la linfadenectomía de Margotini y Bucalossi en 1949 y el uso de la radioterapia a manos de Hirsch y Keynes en 
1924 aumentaron la supervivencia de las pacientes. En la segunda mitad del siglo XX, el uso de quimioterapéuticos, como el tamoxifeno en los 80 y los anticuerpos monoclonales al final del siglo, acompañados del descubrimiento de los genes HER2, BRCA1 y BRCA2 entre 1984 y 1995, impulsaron terapias dirigidas para el tratamiento de este cáncer, mejorando el pronóstico de esta enfermedad. ${ }^{1,2}$

En México, esta patología es la primera causa de muerte por cáncer en mujeres, y la segunda causa de muerte en mujeres. El costo promedio al año por paciente de cáncer de mama es de $\$ 110,459.00$ pesos mexicanos, siendo el diagnóstico tardío una de las causas principales del costo elevado. ${ }^{1,3}$ Las repercusiones no se limitan sólo al sector económico, sino también al social, psicológico, laboral y emocional de la paciente y sus familiares. Debido a esto, es cuantiosa la actualización del personal de salud con la más reciente bibliografía respecto a la epidemiología, clínica, diagnóstico, tratamiento y pronóstico del cáncer de mama; además de compartir en los primeros niveles de atención la necesidad de inicio del tamizaje para detección temprana, para así disminuir el impacto económico, aumentar la esperanza de vida y crear una cultura de prevención. ${ }^{3}$

\section{MATERIAL Y MÉTODOS}

Se realizó una búsqueda en las bases de datos de PubMed, Web of Science y EBSCO usando bibliografía en inglés del año 2015 en adelante con las palabras de búsqueda: breast cancer, pathology, therapy, prevention and control, con el operador booleano "and". Se encontraron un total de 156 artículos. Tras someter dichos textos a los criterios de selección, se obtuvieron 30 artículos. Dichos criterios fueron: artículos originales, completos, con referencias disponibles, publicaciones arbitradas, sin más de cinco años de antigüedad, artículos actualizados en la epidemiología, tamizaje, diagnóstico y tratamiento y que tuvieran un aporte relevante a esta investigación.

\section{RESULTADOS

$$
\text { Epidemiología }
$$

El cáncer de mama es la neoplasia más prevalente en mujeres en todo el mundo, y es de igual manera el cáncer con mayor mortalidad en esta población; en países en vías de desarrollo, el grupo etario con mayor prevalencia es el que abarca los 40-49 años, a diferencia de los países desarrollados, donde la mayor afección se encuentra en mujeres postmenopáusicas, y se presenta en una relación de 100 a 1 entre sexo femenino y masculino. ${ }^{4}$

Aproximadamente, al año se reportan 1.38 millones de nuevos casos y medio millón de defunciones en el mundo derivadas de esta patología. En países desarrollados como Estados Unidos, se ha visto una reducción drástica de la mortalidad en $30 \%$ procedente del aumento de la detección oportuna mediante el uso del tamizaje por mastografía, y el establecimiento de sistemas de detección estandarizados. ${ }^{5}$ Mientras tanto, en países en vías de desarrollo como es el caso de México, se muestra una constate tendencia ascendente (incidencia calculada de 38.4 por 100,000 mujeres y mortalidad estandarizada de 16.8 muertes por 100,000 mujeres), resultado de la poca disponibilidad de instrumentos de tamizaje y métodos de registro del cáncer. ${ }^{4}$

\section{Etiología}

Dentro de la etiopatogenia de esta enfermedad, se sabe que es originada por la interacción de factores genéticos, ambientales y de estilo de vida, como sucede en la mayoría de las neoplasias, mostrando su origen multifactorial. ${ }^{6}$ Basándonos en su origen, existe la siguiente clasificación:

1. Cáncer de mama esporádico: aquel que ocurre en pacientes sin ningún antecedente familiar. Responsable de $70-80 \%$ de los casos.

2. Familiar: en pacientes con un claro historial, pero sin ser atribuible a la alteración de un solo gen, sino a la mutación de diversos genes (herencia multifactorial). Responsable de $15-20 \%$ de los casos.

3. Hereditario: derivado de la mutación de un solo gen, en el ámbito de línea germinal (herencia monogénica). Responsable de 5-10\% de los casos. En este apartado, cerca de $40 \%$ es derivado de mutaciones en el gen BRCA1 y BRCA2., 6

\section{Factores de riesgo y protectores}

El cáncer de mama, al igual que gran parte de las patologías oncológicas, tiene un origen multifactorial. ${ }^{6}$ Entre éstos se incluye: la exposición a radiación, edad mayor a 50 años, sexo femenino, el alto consumo de lípidos, sedentarismo, el consumo de alcohol, la menarca temprana y la menopausia tardía, la nuliparidad, los antecedentes de haber tenido este padecimiento, la inmunosupresión, tabaquismo, las infecciones virales (hepatitis B y Epstein-Barr). ${ }^{7}$ Sin embargo, los factores de riesgo más relacionados son la historia familiar de cáncer de mama, la presencia de genes como el BRCA1 y BRCA2 y la obesidad. ${ }^{8}$

Por otro lado, los principales factores protectores que han sido estudiados son: la lactancia materna (disminuyendo un $4.3 \%$ por cada año de amamantar), los fitoestrógenos (soya, tofu) y la actividad física, aunque son motivo de controversia en la bibliografía actual. ${ }^{9}$ 


\section{Fisiopatología}

En la patogénesis del cáncer de mama se encuentran involucradas diversas vías, como es el caso de la vía fosfatidilinositol 3 kinasa (PI3K/AKT) y la ruta Ras-Raf-MEK-ERK (RAS/MEK/ERK), las cuales se encargan de proteger a las células de la apoptosis; sin embargo, cuando existe alguna mutación en los genes que codifican para estas vías, el mecanismo de apoptosis pierde su funcionalidad. ${ }^{10}$ Se ha podido demostrar que la exposición constante a estrógenos genera estas mutaciones, además de que la sobreexpresión de leptina (asociado a obesidad) en tejido adiposo mamario ocasiona un aumento de la proliferación celular y a la vez la formación de cáncer. ${ }^{4}$

Otras mutaciones asociadas son aquéllas en el guardián del genoma (p53) y del gen asociado a cáncer de mama (BRCA 1 y BRCA 2), las cuales provocan división celular descontrolada, inhibición de apoptosis y metástasis a órganos distantes. ${ }^{11}$

\section{Clasificación}

Las células cancerígenas de mama cuentan con receptores, tanto en la superficie como en su citoplasma y núcleo, los más importantes son los relacionados a estrógeno, progesterona y a la proteína HER2. ${ }^{12}$ De acuerdo con la presencia de estos receptores, el cáncer de mama puede clasificarse de la siguiente manera:

Luminal A: cuenta con receptores positivos para estrógeno y progesterona, tiene buen pronóstico y responde bien a terapia hormonal.

Luminal B: se subdivide en luminal B/HER2 positivo, el cual tiene receptores positivos de progesterona, estrógeno y HER2, tiene peor pronóstico que luminal A y responde bien a inmunoterapia y terapia hormonal. Por el otro lado, está el luminal B/HER2 negativo, que cuenta con receptores positivos para estrógeno y progesterona, pero HER2 negativo se asocia a un riesgo alto de proliferación celular y responde bien a la hormonoterapia.

HER 2+: no cuenta con receptores positivos para estrógeno y progesterona, pero sí para HER2, además de un pronóstico intermedio, responde muy bien a inmunoterapia y con poca respuesta a quimioterapia. Triple negativo: no cuentan con ninguno de los tres receptores, tienden a responder bien a quimioterapia, pero recaen fácilmente. ${ }^{13}$

Histológicamente, el cáncer de mama se puede clasificar en in situ (25\%) e invasivo (75\%). ${ }^{12}$ Entre los in situ se encuentra el carcinoma ductal, el cual es el más común, y carcinoma lobulillar; mientras que los invasivos se clasifican en carcinoma ductal invasivo y lobulillar invasivo. ${ }^{14}$

\section{Cuadro clínico}

Se debe considerar que el cáncer de mama precoz por lo general no causa síntomas, por esto es importante el apoyo con los estudios de imagen e histopatología para diagnóstico y tamizaje. ${ }^{1}$ Los pacientes, en caso de presentar síntomas, pueden referir descamación de la areola y piel, formación de costras, rubor, edema de la mama, formación de hoyuelos cutáneos (piel de naranja), mastalgia, retracción de los pezones, dolor óseo, úlceras cutáneas, adenopatías, pérdida de peso y secreción sanguinolenta. Sin embargo, el síntoma más temprano y frecuente es la aparición de una masa a nivel mamario, no dolorosa, firme y de bordes irregulares. ${ }^{15}$ La presencia de tumoraciones es más frecuente en la región superior lateral (en 50\% de los casos), seguida por la areola en $18 \%$ de los casos.

\section{Diagnóstico}

En la actualidad, existen tres métodos para el abordaje: examen físico y una historia clínica completa, técnicas de imagen y biopsia. ${ }^{4}$

Examen físico e historia clínica: inicialmente, las mujeres de manera mensual deben autoexplorar ambas mamas para que, por medio de visualización y palpación de éstas, se hallen alteraciones que a continuación serán expuestas. La autoexploración ha sido descartada por varias guías internacionales, pero se denota la importancia de que las mujeres conozcan el aspecto de sus mamas para detectar alguna anomalía; en la Guía de Práctica Clínica mexicana se sigue aceptando su uso. ${ }^{16}$ En la consulta médica, la historia clínica se debe indagar de manera detallada sobre factores de riesgo y descartar la presencia de sintomatología mamaria. ${ }^{1}$ Se debe hacer una exploración e interrogatorio detallado y dirigido, para descartar otras patologías diferenciales como fibroadenomas o quistes mamarios. ${ }^{4,16}$

Mamografía y técnicas de imágenes: la mamografía consiste en la obtención de una imagen de la mama tomada con rayos $X$. La sensibilidad de la mamografía (67.8\%) se relaciona con la edad, el origen étnico, la historia personal, además de que es operador dependiente y se requiere que el aparato esté en condiciones adecuadas. ${ }^{17}$ Además existe la mamografía digital, que se basa en la angiogénesis tumoral y también sirve para detectar cáncer de mama, este método se ha utilizado recientemente como la herramienta de elección ideal. ${ }^{16}$ El ultrasonido es un estudio complementario, mas no independiente de la mamografía, y se utiliza para evaluar errores encontrados en ésta, además de abordar a mujeres embarazadas, con implantes mamarios, mamas densas, menores de 35 años o sujetos que no pueden hacerse la mamografía y no es adecuado para estructuras óseas. ${ }^{16}$ Cuenta con una especificidad de $98 \%$. Su limitación principal es que es un 
estudio operador dependiente..$^{18}$ La resonancia magnética es utilizada en mujeres jóvenes con factores de riesgo elevados; como toda técnica, cuenta con limitaciones, las cuales incluyen que algunos tipos de neoplasias no pueden ser detectadas, tal es el caso del ductal y carcinoma lobulillar. Cuenta con una sensibilidad de $70-96 \%$ y una especificidad de $67-100 \%$. ${ }^{4,19}$ La tomografía computarizada se utiliza para determinar la presencia de metástasis, no es un estudio rutinario y tiene indicaciones precisas. Cuenta con una sensibilidad de $91 \%$ y con una especificidad de 93\%. ${ }^{18,19}$ PET (tomografía por emisión de positrones, por sus siglas en inglés) es indicado con base en la terapéutica usada, y se utiliza para la visualización de la metástasis y para definir la respuesta al tratamiento. Cuenta con una sensibilidad de $61 \%$ y con una especificidad de $80 \%{ }^{19}$

Biopsia: Existen varios tipos, como la biopsia por aspiración con aguja fina (aguja pequeña para extraer pocas células), biopsia con aguja de corte (toma una mayor cantidad de tejido, pero para su extracción se necesita el uso de una anestesia local; además puede ser guiada clínica, mastográfica o ultrasonográficamente) y biopsia guiada por imágenes (utiliza la mamografía o el ultrasonido para su realización). ${ }^{20}$

La biopsia es imprescindible para realizar el diagnóstico y la posterior estadificación del cáncer, la cual nos indica el estadio clínico (EC) de la enfermedad y los enfoques terapéuticos y el pronóstico que el paciente tendrá. ${ }^{16}$ Se utiliza la estadificación TNM, diseñada por la American Joint Committee on Cancer (AJCC), donde la "T" hace referencia al tamaño tumoral: T1 (menor o igual a 2 centímetros), T2 (tumor entre 2-5 centímetros), T3 (tumor mayor de 5 centímetros) y T4 (cualquier tamaño, con extensión directa a piel o pared torácica). La "N" representa el compromiso linfático nodal: N0 (sin extensión a ganglios linfáticos, o menores a $0.2 \mathrm{~mm}$ ), $\mathrm{N} 1$ (diseminación de uno a tres ganglios axilares), N2 (diseminación de cuatro a nueve ganglios axilares) y N3 (diseminación a más de 10 ganglios axilares). Por último, la " $\mathrm{M}$ " es utilizada para reportar la metástasis a distancia: M0 (sin enfermedad a distancia) y M1 (con enfermedad a distancia). ${ }^{21}$ De esta estadificación se obtienen los estadios clínicos mencionados en la Tabla 1.

Además, existe una clasificación para el reporte de la mastografía para la posibilidad de malignidad de una lesión hallada, llamado BIRADS (Sistema de Reporte y Datos de Imagen en Mama):

BI-RADS 0: evaluación incompleta.

BI-RADS 1: negativa, mama normal.

BI-RADS 2: hallazgos benignos.

BI-RADS 3: hallazgo probablemente benigno, seguimiento cada seis meses.

BI-RADS 4: anormalidad sospechosa: a) baja sospecha; b) posible sospecha; y c) alta sospecha (indicación de biopsia).
BI-RADS 5: altamente sugestiva de malignidad (indicación de biopsia).

BI-RADS 6: malignidad conocida (confirmada por biopsia). ${ }^{20,21}$

\section{Tamizaje}

La forma más eficaz de reducir la mortalidad debido al cáncer de mama es encontrar el cáncer en una etapa temprana y recibir el tratamiento adecuado para combatir la enfermedad. ${ }^{22}$ La mastografía es la base del tamizaje del cáncer de mama, ya que nos permite detectar tumores antes de que sean palpables o empiece a manifestarse algún síntoma. ${ }^{23}$

Existen diversas guías elaboradas por distintas sociedades, academias y colegios que nos indican las recomendaciones para esta detección temprana del cáncer de mama, tanto para mujeres con riesgo promedio como para aquéllas que tienen un riesgo elevado de padecerlo. ${ }^{22,24}$ Las recomendaciones de las diversas guías internacionales son expuestas en la Tabla 2.

\section{Tratamiento}

El tratamiento para el cáncer de mama se compone principalmente de locales (cirugía y la radioterapia) y terapias sistémicas (quimioterapia, terapia hormonal y terapia dirigida), estas terapias tienen sus indicaciones precisas dependiendo del paciente. La meta final del tratamiento es la curación. ${ }^{25}$

Cirugía: tiene como objetivo eliminar la mayor cantidad de células cancerígenas como sea posible, evaluar

\section{Tabla 1: Estadios clínicos de cáncer} de mama, según su TNM.

Estadio clínico

$\begin{array}{ll}0 & \text { Tis, N0, M0 } \\ \text { I } & \text { IA: T1, N0, M0 } \\ & \text { IB: de TO o T1, N1 mi, M0 } \\ \text { II } & \text { IIA: de T0, N1, M0 a T2, N0, M0 } \\ & \text { IIB: de T2, N1, M0 a T3, NO, M0 } \\ \text { III } & \text { IIIA: de TO, N2, M0 a T3, N2, M0 } \\ & \text { IIIB: de T4, N0 a 2, M0 } \\ & \text { IIIC: T } 1 \text { a 4, N3, M0 } \\ \text { IV } & \text { T1 a 4, N1 a 3, M1 }\end{array}$

TNM = tamaño tumoral, compromiso linfático nodal y metástasis, is = in situ, $\mathrm{mi}=$ micrometástasis (diámetro 0.2 a $2 \mathrm{~mm}$ ).

Fuente: Alamdaran $\mathrm{S}$ et al. ${ }^{20}$ 
Tabla 2: Tamizaje de cáncer de mama por diversas guías internacionales.

\begin{tabular}{|c|c|c|c|c|}
\hline Población & $\begin{array}{c}\text { American Cancer } \\
\text { Society }\end{array}$ & $\begin{array}{l}\text { International Agency for } \\
\text { Research on Cancer }\end{array}$ & $\begin{array}{c}\text { American College of } \\
\text { Physicians }\end{array}$ & $\begin{array}{l}\text { American Academy } \\
\text { of Family Physicians }\end{array}$ \\
\hline $\begin{array}{l}\text { Mujer de } 40 \text { a } 49 \text { años, } \\
\text { riesgo promedio }\end{array}$ & Mastografía anual & Evidencia insuficiente & $\begin{array}{l}\text { Mastografía bienal si } \\
\text { paciente lo solicita }\end{array}$ & $\begin{array}{c}\text { Mastografía si } \\
\text { paciente lo solicita }\end{array}$ \\
\hline $\begin{array}{l}\text { Mujer de } 50 \text { a } 74 \text { años, } \\
\text { riesgo promedio }\end{array}$ & $\begin{array}{c}\text { De } 50 \text { a } 54 \text { años: } \\
\text { mastografía anual } \\
\text { De } 55 \text { años en adelante, } \\
\text { mastografía bienal o anual por } \\
\text { decisión de paciente }\end{array}$ & $\begin{array}{c}\text { De } 50 \text { a } 69 \text { años: } \\
\text { mastografía anual } \\
\text { De } 70 \text { a } 74 \text { años: } \\
\text { mastografía reduce el riesgo } \\
\text { de muerte }\end{array}$ & $\begin{array}{l}\text { Mastografía } \\
\text { bienal }\end{array}$ & $\begin{array}{l}\text { Mastografía } \\
\text { anual }\end{array}$ \\
\hline Mujer, mamas densas & $\begin{array}{c}\text { Evidencia insuficiente para } \\
\text { tamizaje por resonancia } \\
\text { magnética }\end{array}$ & $\begin{array}{c}\text { Evidencia } \\
\text { insuficiente }\end{array}$ & $\begin{array}{l}\text { Evidencia } \\
\text { insuficiente }\end{array}$ & $\begin{array}{l}\text { Evidencia } \\
\text { insuficiente }\end{array}$ \\
\hline Mujer, riesgo elevado & $\begin{array}{l}\text { Mastografía y resonancia } \\
\text { magnética anual }\end{array}$ & $\begin{array}{c}\text { Mastografía y resonancia } \\
\text { magnética debe iniciarse a } \\
\text { edad temprana }\end{array}$ & $\begin{array}{l}\text { Evidencia } \\
\text { insuficiente }\end{array}$ & $\begin{array}{l}\text { Evidencia } \\
\text { insuficiente }\end{array}$ \\
\hline
\end{tabular}

Fuente: Duffy S et al ${ }^{22}$ y Garcia D et al..$^{16}$

la presencia de metástasis y de los ganglios linfáticos, así como restaurar la forma de la mama. ${ }^{22}$ Existen dos tipos de cirugías, la primera es cirugía con conservación de la mama (se extirpa solamente la parte de la mama que tiene cáncer); y la segunda es la mastectomía (se extirpa totalmente la mama e incluso todo el tejido mamario). Se debe hacer énfasis en que una de las partes fundamentales de estos procedimientos es la estadificación axilar. ${ }^{26}$

Radioterapia: tratamiento que usa rayos o partículas de alta energía para eliminar las células cancerígenas. ${ }^{27}$ La utilización de la radioterapia depende de qué tipo de cirugía se realizó, además de si el cáncer involucra metástasis, el tamaño y la afección a la piel. ${ }^{16}$ Los dos tipos principales de radioterapia que se utilizan para el tratamiento del cáncer de mama son radioterapia externa y braquiterapia. ${ }^{4,26}$

Quimioterapia: está dividida en quimioterapia adyuvante y neoadyuvante. La primera hace referencia a la quimioterapia posterior a un tratamiento quirúrgico, con el objetivo de eliminar las células cancerosas que puedan haber permanecido, tiene el objetivo de evitar la recaída. ${ }^{1}$ La segunda hace referencia a la administrada antes de una cirugía o la usada como terapia estándar (en casos donde no puede haber un abordaje quirúrgico). ${ }^{25}$ Dentro de los fármacos más empleados se encuentra la doxorrubicina, epirrubicina, paclitaxel, docetaxel, 5-fluorouracilo, ciclofosfamida y el carboplatino. ${ }^{27}$

Terapia hormonal: este tipo de terapia se utiliza en mujeres que padecen cáncer de mama con receptores positivos (ER-positivo y/o PR-positivo) después de un tratamiento quirúrgico para evitar el riesgo de recaída. ${ }^{27}$ Los medicamentos más empleados en la práctica clínica son el tamoxifeno y los inhibidores de la aromatasa. ${ }^{28}$

Terapia dirigida/biológica: utilizada en los casos donde los medicamentos de quimioterapia no son eficaces, tal es el caso del cáncer de mama HER2 positivo. ${ }^{26}$ El medicamento más usado en esta terapia es el trastuzumab (anticuerpo monoclonal humanizado IgG contra HER2), el cual inhibe la proliferación de células humanas tumorales que sobreexpresan HER2. ${ }^{25}$ Éste puede ser usado antes de la cirugía para tratar el cáncer de mama en etapa inicial, o para tratar el cáncer de mama avanzado. ${ }^{27,28}$

\section{Pronóstico}

El pronóstico de una paciente con cáncer de mama se basa en la edad, la presencia o no de HER-2 (si existe, indica un mejor pronóstico), estadio clínico, grado histológico, el resultado de los microarreglos realizados (como MammaPrint y Oncotype) y con base en las calculadoras de riesgo como adyuvante. ${ }^{29} \mathrm{El}$ punto de inflexión en el pronóstico del cáncer es el diagnóstico temprano, debido a que pacientes con EC I tienen una supervivencia de cinco años de $88 \%$, mientras que un paciente con EC IV tiene una supervivencia aproximada de $15 \%{ }^{30}$ Respecto a la metástasis, los órganos más frecuentes son: hueso, hígado y pulmón. Otros incluyen al cerebro, aunque puede tener potencial de hacer metástasis a cualquier órgano. ${ }^{29}$

En México, las directrices acerca del manejo del cáncer de mama se encuentran en la Norma Oficial Mexicana NOM-041-SSA2-2011 para la prevención, diagnóstico, tratamiento, control y vigilancia epidemiológica del cáncer 
de mama, que establece el tamizaje con mastografía de 40 a 69 años de forma bienal, y para mayores de 70 años dependerá de antecedente personal de este padecimiento o por indicación médica. De igual manera, establece que el diagnóstico se realice de manera histopatológica, y se ha clasificado por medio de las normativas dictadas por el AJCC. Por último, establece cinco caminos de tratamiento: cirugía, radioterapia, quimioterapia, hormonoterapia y biológicos, cada uno con sus indicaciones puntuales. ${ }^{31}$

En conjunto con la Norma Oficial Mexicana, las Guías de Práctica Clínica (GPC) de tratamiento del cáncer de mama en segundo y tercer nivel de atención y la GPC de Prevención, tamizaje y referencia oportuna de casos sospechosos de cáncer de mama en el primer nivel de atención establecen el tamizaje de cáncer de mama en pacientes menores a 40 años dependiendo de factores de riesgo genéticos (si se presentan, se envía a especialista, si no, autoexploración mensual y exploración clínica anual), de 40 a 74 años mastografía bi o trienal, y en mayores de 75 años según su estado actual y esperanza de vida. ${ }^{32}$

\section{DISCUSIÓN Y CONCLUSIONES}

A pesar de que el cáncer de mama es la neoplasia más prevalente en mujeres, se ha visto que los países en vías de desarrollo no han implementado un plan estratégico eficiente, basado en las guías internacionales acerca del tamizaje, para un diagnóstico oportuno del padecimiento, influyendo de manera negativa en el pronóstico. ${ }^{4,5}$ Para lograr una disminución de los casos de cáncer de mama en México, es necesario mejorar las estrategias de detección temprana, junto con una combinación de un abordaje médico integral, para poder hacer frente a este gran desafío. ${ }^{1,3}$

Los estudios actuales han identificado las vías moleculares que son afectadas para el desarrollo de este padecimiento, mostrando su etiología multifactorial. ${ }^{6}$ Estas investigaciones han resultado ser de suma utilidad para el posterior desarrollo de técnicas terapéuticas en los pacientes. ${ }^{25}$

Si bien la mastografía es sin duda el estándar de oro para la detección temprana del padecimiento, se cuenta con diversos estudios de imagen complementarios para confirmar la sospecha diagnóstica, partiendo como siempre de un adecuado interrogatorio y exploración física. ${ }^{16,22}$ Se recalca que una detección temprana es imprescindible para aumentar la esperanza y la calidad de vida. ${ }^{23}$

La capacitación del personal de salud de primer contacto, así como el desarrollo de un plan estratégico eficiente, encaminado a una detección oportuna de esta patología, son de la más alta importancia para generar una cultura de prevención y detección oportuna del cáncer de mama. ${ }^{1,16}$

\section{REFERENCIAS}

1. Murillo R, Díaz S, Perry F, Poveda C, Piñeros M, Sánchez O et al. Increased breast cancer screening and downstaging in Colombian women: A randomized trial of opportunistic breast-screening. Int J Cancer. 2016; 138 (3): 705-713.

2. Brouwers PJAM, van Werkhoven E, Bartelink H, Fourquet A, Lemanski C, van Loon J et al. Predictors for poor cosmetic outcome in patients with early stage breast cancer treated with breast conserving therapy: Results of the Young boost trial. Radiother Oncol. 2018; 128 (3): 434-441.

3. Pistilli B, Mazouni C, Zingarello A, Faron M, Saghatchian M, Grynberg M et al. Individualized prediction of menses recovery after chemotherapy for early-stage breast cancer: a nomogram developed from UNICANCER PACS04 and PACS05 trials. Clin Breast Cancer. 2019; 19 (1): 63-70.

4. Segnan N, Minozzi S, Armaroli P, Cinquini M, Bellisario C, González-Lorenzo $\mathrm{M}$ et al. Epidemiologic evidence of slow growing, nonprogressive or regressive breast cancer: a systematic review. Int J Cancer. 2016; 139 (3): 554-573.

5. Ochalek K, Partsch H, Gradalski T, Szygula Z. Do compression sleeves reduce the incidence of arm lymphedema and improve quality of life? Two-year results from a prospective randomized trial in breast cancer survivors. Lymphat Res Biol. 2019; 17 (1): 70-77.

6. Bartlett JMS, Sgroi DC, Treuner K, Zhang Y, Ahmed I, Piper T et al. Breast Cancer Index and prediction of benefit from extended endocrine therapy in breast cancer patients treated in the Adjuvant Tamoxifen-To Offer More? (aTTom) trial. Ann Oncol. 2019; 30 (11): 1776-1783.

7. Valachis A, Mamounas EP, Mittendorf EA, Hayashi N, Ishitobi M, Natoli $\mathrm{C}$ et al. Risk factors for locoregional disease recurrence after breast-conserving therapy in patients with breast cancer treated with neoadjuvant chemotherapy: An international collaboration and individual patient meta-analysis. Cancer. 2018; 124 (14): 2923-2930.

8. van den Brandt PA, Schulpen M. Mediterranean diet adherence and risk of postmenopausal breast cancer: results of a cohort study and meta-analysis. Int J Cancer. 2017; 140 (10): 2220-2231.

9. Fornusek CP, Kilbreath SL. Exercise for improving bone health in women treated for stages I-III breast cancer: a systematic review and meta-analyses. J Cancer Surviv. 2017; 11 (5): 525-541.

10. Sparano JA, Gray RJ, Ravdin PM, Makower DF, Pritchard KI, Albain KS et al. Clinical and genomic risk to guide the use of adjuvant therapy for breast cancer. N Engl J Med. 2019; 380 (25): 2395-2405.

11. Chan N, Willis A, Kornhauser N, Ward MM, Lee SB, Nackos E et al. Influencing the tumor microenvironment: a phase II study of copper depletion using tetrathiomolybdate in patients with breast cancer at high risk for recurrence and in preclinical models of lung metastases. Clin Cancer Res. 2017; 23 (3): 666-676.

12. Inno A, Barni S, Ghidini A, Zaniboni A, Petrelli F. One year versus a shorter duration of adjuvant trastuzumab for HER2-positive early breast cancer: a systematic review and meta-analysis. Breast Cancer Res Treat. 2019; 173 (2): 247-254.

13. Mittendorf EA, Ardavanis A, Litton JK, Shumway NM, Hale DF, Murray $J L$ et al. Primary analysis of a prospective, randomized, single-blinded phase II trial evaluating the HER2 peptide GP2 vaccine in breast cancer patients to prevent recurrence. Oncotarget. 2016; 7 (40): 66192-66201.

14. Boekhout AH, Gietema JA, Milojkovic Kerklaan B, van Werkhoven ED, Altena R, Honkoop A et al. Angiotensin II-receptor inhibition with candesartan to prevent trastuzumab-related cardiotoxic effects in patients with early breast cancer: a randomized clinical trial. JAMA Oncol. 2016; 2 (8): 1030-1037.

15. Rui L, Guijuan Z, Fengjie B, Min M, Yi M. Eugenol suppresses the development of estrogen receptor-positive precancerous breast lesions and regulates estrogen receptor-related proteins. Acta Medica Mediterr. 2018; 34 (6): 1821-1827. 
16. Garcia D, Spruill LS, Irshad A, Wood J, Kepecs D, Klauber-DeMore $\mathrm{N}$. The value of a second opinion for breast cancer patients referred to a National Cancer Institute (NCl)-designated cancer center with a multidisciplinary breast tumor board. Ann Surg Oncol. 2018; 25 (10): 2953-2957.

17. Soo MS, Jarosz JA, Wren AA, Soo AE, Mowery YM, Johnson KS et al. Imaging-guided core-needle breast biopsy: impact of meditation and music interventions on patient anxiety, pain, and fatigue. J Am Coll Radiol. 2016; 13 (5): 526-534.

18. Lehman CD, Lee JM, DeMartini WB, Hippe DS, Rendi MH, Kalish $G$ et al. Screening MRI in women with a personal history of breast cancer. J Natl Cancer Inst. 2016; 108 (3): djv349.

19. Chen L, Yang Q, Bao J, Liu D, Huang X, Wang J. Direct comparison of $\mathrm{PET} / \mathrm{CT}$ and MRI to predict the pathological response to neoadjuvant chemotherapy in breast cancer: a meta-analysis. Sci Rep. 2017; 7 (1): 8479 .

20. Alamdaran SA, Farrokh D, Tavakoli H, Afzali N, Tavakoli M. Core needle breast biopsy with local dental anesthetics. Breast J. 2017; 23 (6): $772-773$.

21. Foukakis T, von Minckwitz G, Bengtsson NO, Brandberg Y, Wallberg B, Fornander $\mathrm{T}$ et al. Effect of tailored dose-dense chemotherapy vs standard 3-weekly adjuvant chemotherapy on recurrencefree survival among women with high-risk early breast cancer: a randomized clinical trial. JAMA. 2016; 316 (18): 1888-1896.

22. Duffy SW, Vulkan D, Cuckle H, Parmar D, Sheikh S, Smith RA et al. Effect of mammographic screening from age 40 years on breast cancer mortality (UK Age trial): final results of a randomised, controlled trial. Lancet Oncol. 2020; 21 (9): 1165-1172.

23. Knight JA, Blackmore KM, Fan J, Malone KE, John EM, Lynch CF et al. The association of mammographic density with risk of contralateral breast cancer and change in density with treatment in the WECARE study. Breast Cancer Res. 2018; 20 (1): 23.

24. Gao JJ, Tan M, Pohlmann PR, Swain SM. HALT-D: a phase II evaluation of crofelemer for the prevention and prophylaxis of diarrhea in patients with breast cancer on pertuzumab-based regimens. Clin Breast Cancer. 2017; 17 (1): 76-78.

25. Lee O, Ivancic D, Allu S, Shidfar A, Kenney K, Helenowski I et al. Local transdermal therapy to the breast for breast cancer prevention and DCIS therapy: preclinical and clinical evaluation. Cancer Chemother Pharmacol. 2015; 76 (6): 1235-1246.

26. White J, Winter K, Kuske RR, Bolton JS, Arthur DW, Scroggins T et al. Long-term cancer outcomes from study NRG oncology/RTOG 9517: a phase 2 study of accelerated partial breast irradiation with multicatheter brachytherapy after lumpectomy for early-stage breast cancer. Int J Radiat Oncol Biol Phys. 2016; 95 (5): 1460-1465.

27. Coles CE, Griffin CL, Kirby AM, Titley J, Agrawal RK, Alhasso A et al. Partial-breast radiotherapy after breast conservation surgery for patients with early breast cancer (UK IMPORT LOW trial): 5-year results from a multicentre, randomised, controlled, phase 3, noninferiority trial. Lancet. 2017; 390 (10099): 1048-1060.

28. Humbert O, Riedinger JM, Vrigneaud JM, Kanoun S, Dygai-Cochet I, Berriolo-Riedinger A et al. 18F-FDG PET-derived tumor blood flow changes after 1 cycle of neoadjuvant chemotherapy predicts outcome in triple-negative breast cancer. J Nucl Med. 2016; 57 (11): 1707-1712.

29. Baumann R, Dunst J. Prognosis in young women with early-stage breast cancer. Strahlenther Onkol. 2016; 192 (10): 737-738.

30. Arpino G, Pensabene M, Condello C, Ruocco R, Cerillo I, Lauria R et al. Tumor characteristics and prognosis in familial breast cancer. BMC Cancer. 2016; 16 (1): 924.

31. CENETEC [Internet]. NOM-041-SSA2-2011, Para la prevención, diagnóstico, tratamiento, control y vigilancia epidemiológica del cáncer de mama. [Citado 20/10/2020] Disponible en: http://www. cenetec.salud.gob.mx/descargas/equipoMedico/normas/NOM_041_ SSA2 2011.pdf

32. CENETEC [Internet]. Diagnóstico y tratamiento del cáncer de mama en segundo y tercer nivel de atención. México: Secretaría de Salud; 2009. [Citado 20/10/2020] Disponible en: http://dcs.uqroo.mx/ paginas/guiasclinicas/gpc/docs/IMSS-232-09-ER.pdf 